\title{
Appropriate Use Criteria for coronary revascularization in patients with stable ischemic heart disease: What the surgeon needs to know
}

\author{
Harold L. Lazar, MD
}

From the Division of Cardiac Surgery, Boston University School of Medicine, Boston, Mass.

Disclosures: Author has nothing to disclose with regard to commercial support.

Received for publication April 28, 2017; accepted for publication May 11, 2017; available ahead of print June 9, 2017

Address for reprints: Harold L. Lazar, MD, Division of Cardiac Surgery, Boston University School of Medicine, 88 E Concord St, Boston, MA 02118 (E-mail: Harold.l.lazar@gmail.com).

J Thorac Cardiovasc Surg 2019;157:144-6

$0022-5223 / \$ 36.00$

Copyright (c) 2017 by The American Association for Thoracic Surgery

http://dx.doi.org/10.1016/j.jtcvs.2017.05.029

In 2009, the American College of Cardiology in collaboration with other professional societies, including the American Association for Thoracic Surgery, Society of Thoracic Surgeons (STS), and American Heart Association, published the first Appropriate Use Criteria (AUC) for coronary revascularization. ${ }^{1}$

As opposed to the usual clinical guidelines, in which a Class I Recommendation is "always beneficial," Class II is divided into "A-may be beneficial" and "B-may or may not be beneficial," and Class III is "harmful-should not be performed," the original AUC consisted of "appropriate for revascularization," "inappropriate," or "uncertain." In 2013, the terminology was changed to its current definitions: "appropriate care," "may be appropriate care," and "rarely appropriate care." Thus, no intervention is considered "harmful" or "should not be performed."

In previous AUC documents, indications for revascularization for acute coronary syndrome (ACS) and stable ischemic heart disease (SIHD) were combined into 1 document. As opposed to the AUC for ACS, ${ }^{2}$ the AUC on SIHD ${ }^{3}$ review clinical scenarios in which coronary artery bypass grafting (CABG) plays an important role and is the subject of this expert commentary.

In the current AUC for SIHD, an independent rating panel consisting of 5 interventional cardiologists, 6 noninterventional cardiologists, 5 cardiac surgeons, and 1 outcomes researcher scored various clinical scenarios designed to mimic events encountered in the everyday practice of patients with ischemic heart disease. This included data regarding symptoms, noninvasive testing, coronary artery disease burden as assessed by angiography and Synergy Between Percutaneous Coronary Intervention with Taxus and Cardiac Surgery (SYNTAX) scores, fractional flow reserve, and the presence or absence of diabetes. Comorbidities that were not assessed, but are present in the STS scoring system, included chronic kidney disease, reduced ejection fraction, peripheral vascular disease, and chronic obstructive pulmonary disease. Each clinical

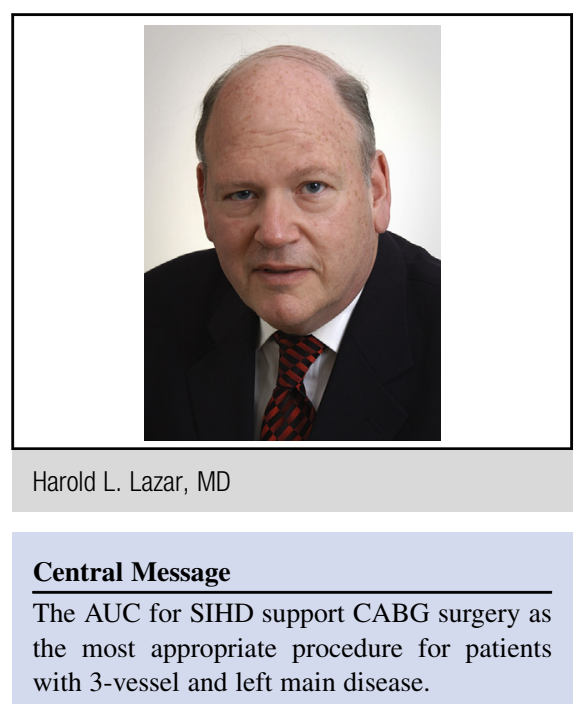

See Article by Patel et al. scenario was rated on a scale of 1 to 9 , in which 7 to 9 indicated that revascularization was "appropriate," 4 to 6 indicated that revascularization "may be appropriate," and 1 to 3 indicated that revascularization "is rarely appropriate." The current AUC for SIHD differed from previous documents in that methods to determine the clinical significance of coronary stenosis using fractional flow reserve were now included. The role of antianginal therapy was expanded to better access the quality of medical care, and the AUC now included patients being considered for coronary revascularization who were being evaluated for renal transplant and percutaneous valve procedures.

In general, the ratings assigned for surgical revascularization by the AUC are "appropriate" and consistent with current society guidelines for $\mathrm{CABG}$ surgery. Patients with a low burden of coronary artery disease such as singlevessel disease, patients with a low risk for ischemia on noninvasive testing, and patients receiving no antianginal therapy are "rarely appropriate" for CABG or percutaneous coronary interventions (PCIs). CABG is "appropriate" for symptomatic 3-vessel disease and 1and 2-vessel disease unresponsive to medical therapy, asymptomatic 3-vessel disease with strongly positive image testing, all left main lesions, and 3-vessel disease with SYNTAX scores greater than 22. CABG also is appropriate for reoperative surgery in the absence of a patent left internal 
thoracic artery to the left anterior descending artery or unrevascularized left anterior descending artery disease and in those reoperative surgical patients with patent left internal thoracic artery but who have other unrevascularized stenotic vessels with persistent angina symptoms despite medical therapy. CABG also is considered "appropriate" for potential renal transplant recipients with extensive 3vessel disease or left main lesions. What is different in the current AUC guidelines is that now PCI "may be appropriate" for 3-vessel disease and SYNTAX scores greater than 22; "may be appropriate" for ostial and mid shaft left main lesions with SYNTAX scores greater than 22; and "may be appropriate" for bifurcation left main lesions and SYNTAX scores less than 22. PCI is now "appropriate" for renal transplant recipients and patients with percutaneous valve and mitral clip with left main lesions, and 3-vessel disease with SYNTAX scores greater than 22 .

What is the role of the AUC in the practice of SIHD and how has it affected clinical practice? As a result of the AUC, third-party payers began to incorporate measures of PCI appropriateness to reduce inappropriate PCIs and use AUC for pay-for-performance programs. ${ }^{4,5}$ New York State proposed to base PCI reimbursement on AUC and adopted a policy that PCI performed in Medicaid patients who were classified as "inappropriate" would no longer be reimbursed. ${ }^{6}$ However, this policy was abandoned in 2016, and no payment was ever denied. However, those changes had a dramatic effect on the practice of PCI in New York State. Between 2010 and 2014, the percentage of inappropriate PCI in New York State for patients without ACS or prior CABG surgery decreased from $18.2 \%$ to $10.6 \%$ for all patients and from $15.3 \%$ to $6.8 \%$ for Medicaid patients. ${ }^{7}$ Appropriate PCIs among Medicaid patients decreased from 340 in 2010 to 84 in 2014, a $75 \%$ decrease. The total number of stable patients receiving PCI rated as "inappropriate" decreased from 2956 in 2010 to 911 in 2014 , a decrease of $69.2 \%$. Furthermore, there was a substantial decrease in the number of stable patients undergoing PCI. This was consistent with national trends showing that the volume of nonacute PCIs declined by $34 \%$, with the percentage of cases classified as "inappropriate" decreasing by $50 \%{ }^{8}$ This shift in the practice of PCI may reflect better patient selection or merely the reluctance of interventional cardiologists not to perform procedures that would not get reimbursed. However, it also could reflect "gaming" of the system by "upcoding" clinical symptoms so that patients would be classified as having an ACS when their PCI was performed.

How will the new AUC affect the practice of CABG surgery? As noted previously, the current AUC support the traditional indications for CABG in patients with SIHD. However, concerns should be raised that although the new criteria resulted in PCI procedures receiving lower scores than $\mathrm{CABG}$ for traditional $\mathrm{CABG}$ indications, they are still described as "may be appropriate" and "rarely appropriate" instead of "may not be beneficial" or "harmful." This will allow PCI to be performed for 3-vessel and left main disease. Ultimately, this could result in a decrease in the volume of CABG surgery. It will be more difficult for third-party payors to deny payment for a procedure that "may be appropriate" or "is rarely appropriate" as opposed to one that is termed "inappropriate," "uncertain," or "harmful." More important, extending the appropriateness criteria for PCI to patients with left main and 3-vessel disease, regardless of their SYNTAX score, ultimately increases the long-term morbidity and mortality for these patients. Previous studies have shown that performing CABG surgery after multivessel PCI increases morbidity and mortality, especially in diabetic patients. ${ }^{9}$ Most studies showing noninferiority between PCI and CABG in patients with left main or 3-vessel disease have only 1- to 3-year follow-up. This has been proved to be an inadequate time period to assess future major adverse events. This was demonstrated in the recent NOBLE trial that looked at CABG versus $P C I$ in patients with unprotected left main stenosis. ${ }^{10}$ After 5 years, CABG was found to be superior to PCI, which was driven primarily by a higher incidence of repeat revascularization and myocardial infarctions in the PCI group. Likewise, in the EXCEL trial, which looked at CABG versus PCI in patients with left main disease and low and intermediate SYNTAX scores, although PCI was noninferior to $\mathrm{CABG}$ after 3 years in regard to the composite end points of death, stroke, or myocardial infarction, the PCI group exhibited a greater increase in adverse events between 30 days and 3 years compared with the patients undergoing CABG $(11.5 \%$ vs $7.9 \% ; P=.02) .{ }^{11}$ This suggests that when the data are reviewed after 5 years, CABG may be the superior revascularization procedure for these patients.

How can surgeons use the AUC to support the appropriate use of both CABG and PCI procedures? One of the goals of the AUC was to identify those instances when PCI or other forms of revascularization may have been overused. This was hoped to provide a mechanism for reducing the "overuse" of revascularization procedures. Societies such as the STS, American Association for Thoracic Surgery, American College of Cardiology, and American Heart Association should monitor the incidence of those procedures that have been rated by the AUC as "rarely appropriate" and "may be appropriate" to identify those practitioners or institutions whose use of procedures that fall into these categories exceeds certain predetermined "normal values." This would be similar to maintaining state and national databases designed to monitor institutional and individual mortality in patients undergoing CABG. Although the AUC guidelines emphasize that an "appropriate" care rating does not imply that a specific type of revascularization procedure (CABG or PCI) should be performed or that a rating of "rarely appropriate" should 
preclude a revascularization procedure, it does stipulate that in those instances when the AUC rating is not followed, there should be sufficient documentation as to why an alternative therapy was performed. In those instances when a procedure is performed for a "rarely appropriate" indication, additional documentation would be necessary to justify whether the procedure should be reimbursed. Although heart teams may play an important role in helping to decide what therapy is most appropriate, as in the case with percutaneous valves, third-party payors ultimately may be the final arbiter to determine the most appropriate revascularization strategy. It also may be that procedures performed for "may be appropriate" or "rarely appropriate" may not be reimbursed at the same rate as those for "appropriate" indications. Finally, it is undecided as to how "patient preference" will be factored into this reimbursement equation. What should be done for those patients who require a procedure that is rated "appropriate" but insists on having one that is rated "may be appropriate" or "rarely appropriate"? A graded level of reimbursement for the practitioner with a larger copay for the patient may be the "appropriate" solution in such cases.

The current AUC for SIHD may be seen as a glass that is half full or half empty by cardiac surgeons. The AUC rankings still support $\mathrm{CABG}$ as the most appropriate procedure for patients with extensive multivessel and left main disease. Surgeons should continue to insist that there be close monitoring so there is adherence to AUC guidelines to improve "appropriate" patient selection for coronary revascularization and minimize the overuse of inappropriate revascularization procedures.

\section{References}

1. Patel MR, Dehmer GJ, Hirshfeld JW, Smith PK, Spertus JA. ACCF/SCA/STS/ AATS/AHA/ASNC 2009 appropriateness criteria for coronary revascularization: a report of the American College of Cardiology Foundation Appropriateness Criteria Task Force, Society for Cardiovascular Angiography and Interventions,
Society of Thoracic Surgeons, American Association for Thoracic Surgery, American Heart Association, and the American Society of Nuclear Cardiology. J Am Coll Cardiol. 2009;53:530-3.

2. Patel MR, Calhoon JH, Dehmer GJ, Grantham JA, Maddox TM, Maron DJ, et al. ACC/AATS/AHA/ASE/ASNC/SCAI/SCCT/STS 2016 Appropriate Use Criteria for coronary revascularization in patients with acute coronary syndromes. $J$ Am Coll Cardiol. 2017;69:570-91.

3. Patel MR, Calhoon JH, Dehmer GJ, Grantham JA, Maddox TM, Maron DJ, et al. ACC/AATS/AHA/ASE/ASNC/SCAI/SCCT/STS 2017 appropriate use criteria for coronary revascularization in patients with stable ischemic heart disease: a report of the American College of Cardiology Appropriate Use Criteria Task Force, American Association for Thoracic Surgery, American Heart Association, American Society of Echocardiography, American Society of Nuclear Cardiology, Society for Cardiovascular Angiography and Interventions, Society of Cardiovascular Computed Tomography, and Society of Thoracic Surgeons. J Am Coll Cardiol. 2017;69:2212-41.

4. Society for Cardiovascular Angiography and Interventions. Five Things Physicians and Patients Should Question. American Board of Internal Medicine Choosing Wisely Website. Available at: http://www.choosingwisely.org/ societies/society-for-cardiovascular-angiography-and-interventions/ Accessed December 20, 2016.

5. Blue Cross Blue Shield of Michigan Value Partnerships. Quality Collaborations: Angioplasty. Available at: http://www.valuepartnerships.com/vp-collab/ angioplasty/. Accessed December 20, 2016.

6. New York State Department of Health Medicaid Redesign Team. Basic Benefit Review Work Group. Final Recommendations. November 1, 2011. Available at: https://www.health.ny.gov/healthcare/medicaid/redesign/docs/basic-benefitreview-wrk-grp-final-rpt.pdf. Accessed December 22, 2016.

7. Hannan EL, Samadashuili Z, Cozzens K, Gesten F, Osinaga A, Fish DG, et al. Changes in percutaneous coronary interventions deemed "inappropriate" by Appropriate Use Criteria. J Am Coll Cardiol. 2017;69: 1234-42.

8. Desai NR, Bradley JM, Parzynski CS. Appropriate Use Criteria for coronary revascularization and trends in utilization, patient selection, and appropriateness of percutaneous coronary intervention. JAMA. 2015;314: 2045-53.

9. Lazar HL. Detrimental effects of coronary stenting on subsequent coronary artery bypass graft surgery. Is there another red flag on the field? J Thorac Cardiovasc Surg. 2009;138:276-7.

10. Makikallio T, Holm NR, Lindsay M, Spence MS, Erglis A, Menown IB, et al. Percutaneous coronary angiography versus coronary artery bypass grafting in treatment of unprotected left main stenosis (NOBLE): a prospective, randomized, open-label, non-inferiority trial. Lancet. 2016;388: 2743-52.

11. Stone GW, Sabik JF, Serruys PN, Simonton CA, Genereux P, Puskus J, et al. Everolimus-eluting stents or bypass surgery for left main coronary artery disease. N Engl J Med. 2016;375:2223-35. 